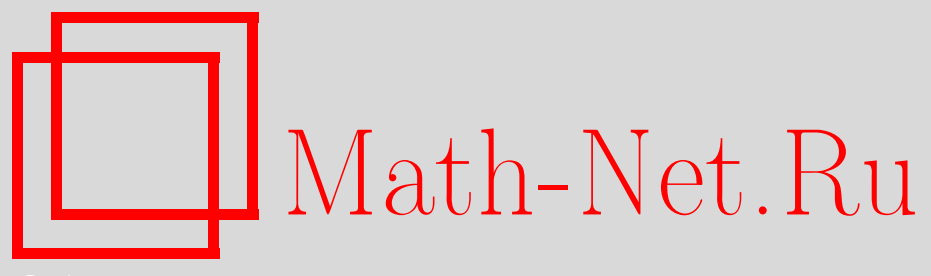

Н. В. Миллионщиков, Сходимость почти наверное ядерных оценок плотности для слабо зависимых случайных полей, УМН, 2006, том 61, выпуск 1, 181-182

DOI: https://doi.org/10.4213/rm1694

Использование Общероссийского математического портала Math-Net.Ru подразумевает, что вы прочитали и согласны с пользовательским соглашением http://www . mathnet.ru/rus/agreement

Параметры загрузки:

IP: 54.157 .27 .8

26 апреля 2023 г., 14:09:31

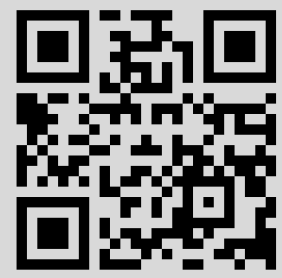




\section{Сходимость почти наверное ядерных оценок плотности для слабо зависимых случайных полей}

\section{Н. В. Миллионщиков}

Задача оценивания плотности распределения случайных величин является одной из основных задач статистического анализа данных. Большое количество работ посвящено изучению свойств ядерных оценок плотности (см., например, [1]-[3]).

Пусть $X_{i}, i \in U_{n},-$ одинаково распределенные случайные векторы со значениями в $\mathbb{R}^{s}$, имеющие общую плотность распределения $f$, а $U_{n}$ - конечное подмножество $\mathbb{Z}^{d}$, $d \in \mathbb{N}$. Ядерная оценка, или оценка Парзена-Розенблатта, плотности $f$ задается формулой

$$
\widehat{f}_{n}(x):=\left|U_{n}\right|^{-1} h_{n}^{-s} \sum_{i \in U_{n}} K\left(\frac{x-X_{i}}{h_{n}}\right), \quad x \in \mathbb{R}^{s}, \quad n \in \mathbb{N},
$$

где $K$ - плотность некоторого вероятностного распределения, $\left\{h_{n}\right\}_{n \geqslant 1}$ - последовательность положительных чисел, $\lim _{n \rightarrow \infty} h_{n}=0$, a $\left|U_{n}\right|$ - число элементов множества $U_{n}$.

Состоятельность оценок (1) изучалась в случае, когда величины $X_{i}$ независимы или удовлетворяют некоторым условиям зависимости, например, условиям перемешивания, положительной (отрицательной) ассоциированности (см., например, [2] и [3]). В данной работе используется подход к описанию стохастической зависимости, предложенный в [4] и [5]. Далее нам потребуются некоторые понятия и обозначения.

Функция $f: \mathbb{R}^{s} \rightarrow \mathbb{R}$ называется липшицевой, если

$$
\operatorname{Lip}(f)=\sup _{x, y \in \mathbb{R}^{s}, x \neq y}|f(x)-f(y)| /\|x-y\|_{1}<\infty,
$$

где $\|x\|_{1}=\sum_{i=1}^{s}\left|x_{i}\right|, x=\left(x_{1}, \ldots, x_{s}\right) \in \mathbb{R}^{s}$. Класс ограниченных липшицевых функций $f: \mathbb{R}^{s} \rightarrow \mathbb{R}$, где $s \in \mathbb{N}$, обозначим $B L$.

Определим функционал $\psi: B L^{2} \times \mathbb{N}^{2} \rightarrow \mathbb{R}$ и для $I \subset \mathbb{Z}^{d}, r \in \mathbb{N}^{d}$ множество $I(r) \subset \mathbb{R}^{d}$ следующим образом:

$$
\psi(f, g, x, y):=\min (x, y) \operatorname{Lip}(f) \operatorname{Lip}(g), \quad I(r):=\bigcup_{i \in I} \prod_{k=1}^{d}\left(i_{k}-r_{k}, i_{k}+r_{k}\right) .
$$

ОПредЕлениЕ. Пусть $U \subseteq \mathbb{Z}^{d}, d \geqslant 1$. Случайное поле $X=\left\{X_{t}, t \in U\right\}$ со значениями в $\mathbb{R}^{s}, s \geqslant 1$, называется $(B L, \psi, \theta)$-зависимым, где $\theta$ - массив положительных чисел $\theta=\left\{\theta_{r}\right\}, r \in \mathbb{N}^{d}$, если для любых непересекающихся конечных множеств $I, J \subset U$ с $I(r) \cap J=\varnothing$ и всех ограниченных липшицевых функций $f: \mathbb{R}^{s|I|} \rightarrow \mathbb{R}$ и $g: \mathbb{R}^{s|J|} \rightarrow \mathbb{R}$

$$
\left|\operatorname{cov}\left(f\left(X_{i}, i \in I\right), g\left(Y_{j}, j \in J\right)\right)\right| \leqslant \psi(f, g,|I|,|J|) \theta_{r} .
$$

Заметим, что в этом определении заложена возможность анизотропного изменения зависимости поля $X$. Для обширного класса случайных процессов и полей оно обобщает понятия положительной и отрицательной ассоциированности (см. [5]).

В [3] доказано, что при весьма широких условиях $\left|\widehat{f}_{n}(x)-\mathrm{E} \widehat{f}_{n}(x)\right|=O\left(\left(n h_{n}\right)^{-\gamma / 2}\right)$ п.н. для всех $\gamma \in(0,1)$. При этом, в частности, предполагается, что $\left\{X_{i}\right\}_{i \geqslant 1}$ образуют строго стационарную последовательность положительно или отрицательно ассоциированных случайных величин и $\sum_{|j| \geqslant m} \operatorname{cov}\left(X_{1}, X_{j+1}\right) \leqslant \alpha^{-m}$ для некоторого $\alpha>1$ и всех $m \in \mathbb{N}$. Сходные результаты для последовательностей ассоциированных случайных величин получены в [2].

Работа выполнена при поддержке РФФИ (грант № 03-01-00724). 
В отличие от [2] и [3] мы рассматриваем серии зависимых случайных полей $X^{(n)}=$ $\left\{X_{i}^{(n)}, i \in U_{n}\right\}$ с векторными значениями. Для них удается получить более точную оценку скорости сходимости $\widehat{f}_{n}(x)$ к $f(x)$, чем в упомянутых работах [2], [3]. При каждом $n \in \mathbb{N}$ оценки $\widehat{f}_{n}(x)$ строятся по элементам полей $X^{(n)}$ в соответствии с формулой (1).

Введем ряд требований.

$1^{\circ} .\left\{U_{n}\right\}_{n \in \mathbb{N}}-$ последовательность конечных подмножеств $\mathbb{Z}^{d}$. При всех $n \in \mathbb{N}$ случайные поля $X^{(n)}$ являются $\left(B L, \psi, \theta^{(n)}\right)$-зависимыми, случайные векторы $X_{i}^{(n)}$ имеют общую плотность распределения $f$, удовлетворяющую условию Липшица, а для $i \neq j$ случайные векторы $\left(X_{i}^{(n)}, X_{j}^{(n)}\right)$ обладают плотностью распределения, ограниченной равномерно по $n, i, j$.

$2^{\circ}$. Ядро $K$ удовлетворяет условию Липшица и $\int_{\mathbb{R}^{s}}\|x\| K(x) d x<\infty$.

Введем $\sigma_{f}^{2}:=f(y) \int_{x \in \mathbb{R}^{s}} K^{2}(x) d x>0$. Из $2^{\circ}$ следует, что $\sup _{x \in \mathbb{R}^{s}} K(x)=M<\infty$.

$3^{\circ}$. Существуют последовательности $\left\{p_{n}\right\}_{n \in \mathbb{N}},\left\{q_{n}\right\}_{n \in \mathbb{N}}$ элементов $\mathbb{N}^{d}$ такие, что $p_{n 1} \cdots p_{n d} \leqslant C_{1}\left(\left|U_{n}\right| h_{n}^{s} / \ln n\right)^{1 / 2}$ для всех достаточно больших $n \in \mathbb{N}$, где $C_{1}<\sigma_{f} / M$ и $\lim _{n \rightarrow \infty} q_{n 1} \cdots q_{n d} h_{n}^{s}=0$. Кроме того, для некоторых $C_{2} \geqslant 0, C_{3}>M / \sigma_{f}$ справедливо неравенство $\theta_{p_{n}}^{(n)} \leqslant C_{2} \exp \left\{-C_{3} \sqrt{\left|U_{n}\right| h_{n}^{-s} \ln n}\right\}$. Пусть также $\lim _{n \rightarrow \infty} h_{n}^{-(s+2)} \theta_{q_{n}}^{(n)}=0$

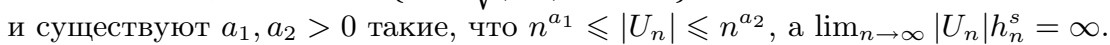

$4^{\circ}$. Плотность $f$ дважды непрерывно дифференцируема, $\sup _{x \in \mathbb{R}^{s}}\left|\frac{\partial f(x)}{\partial x_{i} \partial x_{j}}\right|<C_{4}$ для всех $i, j=1, \ldots, s$ и некоторого $C_{4}>0, \int_{x \in \mathbb{R}^{s}} x K(x) d x=0$ и $\int_{x \in \mathbb{R}^{s}}\|x\|^{2} K(x) d x<\infty$.

$\mathrm{C}$ помощью новых экспоненциальных неравенств для распределений сумм зависимых случайных величин установлена следующая теорема.

Теорема. Пусть выполняются условия $1^{\circ}, 2^{\circ}, 3^{\circ}$ и либо $\lim _{n \rightarrow \infty}\left|U_{n}\right| h_{n}^{s+2}=0$, либо верно условие $4^{\circ} u \lim _{n \rightarrow \infty}\left|U_{n}\right| h_{n}^{s+4}=0$. Тогда для всех $x \in \mathbb{R}^{s}$ таких, ито $f(x)>0,\left|\widehat{f}_{n}(x)-f(x)\right|=O\left(\sqrt{\left|U_{n}\right|^{-1} h_{n}^{-s} \ln \left|U_{n}\right|}\right)$ п.н. при $n \rightarrow \infty$.

СледствиЕ. Пусть выполняются условия $1^{\circ}, 2^{\circ} u 4^{\circ}$. Предположим, что $h_{n} \sim$ $\left|U_{n}\right|^{-\alpha / s}$ для некоторого $\alpha \in(s /(s+4), 1) u \theta_{r}^{(n)} \leqslant \exp \left\{-m_{r}^{d \frac{1+\alpha+\delta}{1-\alpha-\delta}}\right\}$ для всех достаточно больших $n \in \mathbb{N}$ и любых $r \in \mathbb{N}^{d}$, здесь $\delta \in(0,1-\alpha)$, а $m_{r}=\min _{i=1, \ldots, d}\left|r_{i}\right|$. Если существуют $a_{1}, a_{2}>0$ такие, что $n^{a_{1}} \leqslant\left|U_{n}\right| \leqslant n^{a_{2}}$, то при всех $x \in \mathbb{R}^{s}$, для которых $f(x)>0$, имеем $\left|\widehat{f}_{n}(x)-f(x)\right|=O\left(\left|U_{n}\right|^{-(1-\alpha) / 2}\left(\ln \left|U_{n}\right|\right)^{1 / 2}\right)$ п.н. при $n \rightarrow \infty$.

Заметим, что если использовать экспоненциально быстрое убывание $\theta_{r}$ вместо сверхэкспоненциального, то для полей $X^{(n)}$ справедлив аналог результатов, полученных в [3] для процессов.

\section{Список литературы}

[1] А.В. Булинский, Н.В. Миллионщиков, Теория вероятн. и матем. статист., 66 (2002), 34-45. [2] C. Henriques, P. E. Oliveira, "Almost optimal convergence rates for kernel density estimation under association”, Preprint № 04-06, Departamento de Matemática, Universidade de Coimbra, 2002. [3] E. Masry, Statist. Probab. Lett., 58:2 (2002), 205-219. [4] P. Doukhan, S. Louhichi, Stochastic Process. Appl., 84:2 (1999), 313-342. [5] A. Bulinski, Ch. Suquet, Statist. Probab. Lett., 54:2 (2001), 215-226.

Н. В. Миллионщиков (N. V. Millionshchikov)

Московский государственный университет им. М. В. Ломоносова
Представлено А. В. Булинским Принято редколлегией 01.12 .2005 